\title{
Particle-in-cell simulation of coherent and superradiant Smith-Purcell radiation
}

\author{
D. Li, ${ }^{1}$ Z. Yang, ${ }^{2,3}$ K. Imasaki, ${ }^{1}$ and Gun-Sik Park ${ }^{2}$ \\ ${ }^{1}$ Institute for Laser Technology, 2-6 yamada-oka, suita, Osaka 565-0871, Japan \\ ${ }^{2}$ School of Physics, Seoul National University, Seoul 151-747, Korea \\ ${ }^{3}$ Institute of High-Energy Electronics, University of Electronic Science and Technology of China, \\ Chengdu, 610054, People's Republic of China \\ (Received 24 November 2005; published 11 April 2006)
}

\begin{abstract}
This paper presents a study of coherent and superradiant Smith-Purcell (SP) radiation with the help of a two-dimensional particle-in-cell (PIC) simulation. The simulation model supposes a rectangular grating with period length of $173 \mu \mathrm{m}$ to be driven by a single electron bunch, a train of periodic bunches and a continuous beam, respectively. We chose $40 \mathrm{keV}$ as the initial energy of electrons and therefore the SP radiation frequency falls in the $\mathrm{THz}$ regime. From our single bunch simulation we distinguish the true SP radiation separated in time from the emission of the evanescent wave. The evanescent wave radiates from both ends of the grating and is characterized by an angle independent frequency lower than the minimum allowed SP frequency. In order to avoid the buildup of beam bunching from an initially continuous beam, we use a train of periodic bunches to excite the grating and observe the superradiant phenomenon. The repetition frequency of the spatially periodic bunches is assumed to be $300 \mathrm{GHz}$. We find that the superradiant radiation is only emitted at higher harmonics of this frequency and the corresponding SP angles. This result conforms to the viewpoint of Andrews and co-workers. The simulation with a continuous beam shows the dependence of the output power on the beam current. The power curve shows two regimes, one for the incoherent SP radiation and the other for the superradiance, which resembles the Dartmouth experimental result. And furthermore, the frequency spectrum shows an apparent difference for the two regimes, which is in contrast to the observations of Urata and co-workers.
\end{abstract}

DOI: 10.1103/PhysRevSTAB.9.040701

PACS numbers: 41.60.Cr, 52.65.Rr, 07.57.Hm

\section{INTRODUCTION}

A renewed interest in SP radiation has been raised in recent years, since Urata and co-workers observed the superradiance in the $\mathrm{THz}$ regime from the experiment at Dartmouth college [1,2]. The $\mathrm{THz}$ sources, a currently active research area, are of importance in a variety of applications to biophysics, medical, and materials science $[3,4]$. The superradiant SP radiation is a promising alternative in the development of a compact, tunable, and high power $\mathrm{THz}$ device. To improve the performance of such kinds of devices, it is necessary to understand in detail how the coherent and superradiant SP radiation takes place.

It is well known that the SP radiation is emitted when an electron passes near the surface of a periodic metallic grating [5]. The wavelength $\lambda$ of the radiation observed at the angle $\theta$ measured from the direction of electron beam is given by

$$
\frac{\lambda}{L}=\frac{1}{|n|}\left(\frac{1}{\beta}-\cos \theta\right)
$$

where $L$ is the grating period, $\beta c$ the electron velocity, $c$ the speed of light, and $n$ the order of the reflection from the grating. The incoherent SP radiation has been analyzed in many ways, using diffraction theory, integral equation method, and induced surface current model [6-12]. The superradiant radiation of the Dartmouth experiment is regarded as the result of electron beam bunching, induced by the strong interaction of the continuous beam with the evanescent wave propagating along the grating surface. Several theories have been proposed to explain the superradiant phenomenon and to calculate the growth rate of the radiation [13-16], but these analytical results are not in agreement with each other. Some details about the difference can be found in Ref. [15].

Recently, Kesar et al. reported the first simulation of SP radiation [17]. Their simulation model employs a point charge to drive a grating and hence cannot provide any information on the superradiant effect. More recently, Donohue and Gardelle employed a PIC code and performed the simulation in the few $\mathrm{GHz}$ regime with a continuous electron beam [18]. They carefully studied the electron-wave interaction and demonstrated the beam bunching and the superradiant radiation. They also addressed the relation of growth rate and beam current. However, their simulation concerning the output power is insufficient, e.g., they did not provide the dependence of output power on the beam current, which is an important result of the Dartmouth experiment.

In this paper, with the help of a two-dimensional PIC simulation, we study the problems related to the coherent and superradiant SP radiation. The simulation for a single electron bunch is carried out first, from which we can observe the process of generation of the SP radiation and the evanescent wave. Then, we simulate a train of periodic bunches to demonstrate the properties of the superradiant radiation. The advantage of using a prebunched beam is that, the buildup of beam bunching from an initially con- 
tinuous beam is avoided. Finally the simulation with a continuous beam is performed. We find the relation between output power and the beam current, which is not given in Ref. [18].

\section{SIMULATION DESCRIPTION}

The PIC code employed in our simulation, MAGIC [19], is developed by Mission Research Corporation. It is a finite-difference, time-domain code for simulating processes that involve interactions between space charge and electromagnetic fields. We notice that Donohue and Gardelle used the same code in their work.

The simulation geometry is shown in Fig. 1. A grating with rectangular form is set in the center of the bottom of the simulation box. The surface of the grating is assumed to consist of a perfect conductor whose grooves are parallel and uniform in the $z$ direction. We use a sheet electron beam with thickness of $24 \mu \mathrm{m}$, and place its edge $34 \mu \mathrm{m}$ above the top of the grating. It is a perfect laminar beam produced by the MAGIC algorithm and is generated from a cathode located at the left boundary of the simulation box. The electron-wave interaction and radiation propagation happen in the vacuum area, which is enclosed by a special region (called free space in MAGIC language), where the incident electromagnetic waves and electrons can be absorbed. The whole simulation area is divided into a mesh with rectangle cells of small size $(\delta x=17.3 \mu \mathrm{m}, \delta y=$ $17.3 \mu \mathrm{m})$ in the region of beam propagation and grating, and large size $(\delta x=17.3 \mu \mathrm{m}, \delta y=51.9 \mu \mathrm{m})$ in the rest of the region. The Cartesian coordinate system is adopted with the origin at the center of the grating. Since it is a twodimensional simulation, it assumes that all fields and currents are independent of the $z$ coordinate. And it should be noted that the current value mentioned in this paper represents the current per meter in the $z$ direction.

The main parameters of the grating and electron beam are summarized in Table I. The electron beam specification will be varied to match the simulation of a single bunch, a

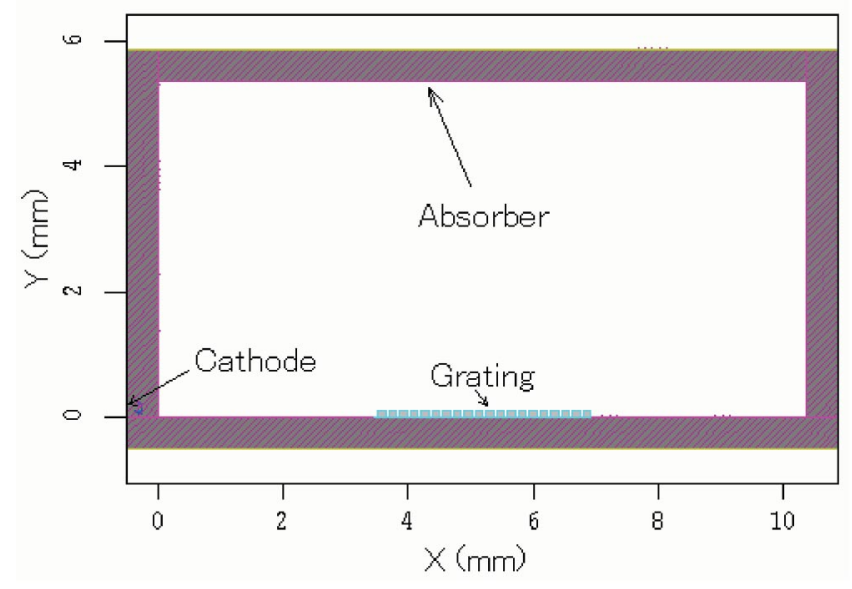

FIG. 1. (Color) Geometry used in simulation.
TABLE I. Main parameters for simulation.

\begin{tabular}{lc}
\hline \hline Grating period & $L=173 \mu \mathrm{m}$ \\
Groove width & $w=62 \mu \mathrm{m}$ \\
Groove depth & $d=100 \mu \mathrm{m}$ \\
Electron beam energy & $E=40 \mathrm{KeV}$ \\
Beam thickness & $\sigma=24 \mu \mathrm{m}$ \\
Beam-grating distance & $\delta=34 \mu \mathrm{m}$ \\
External magnetic field & $B_{x}=2 T$ \\
\hline \hline
\end{tabular}

train of bunches and a continuous beam, respectively. And it will be described in detail for each case when necessary. The external magnetic field is only used for the continuous beam simulation, in order to ensure stable beam propagation above the grating. It should be noted that some parameters of the grating and electrons, such as period length, groove depth and width, and electron's energy, used in our simulation are the same as those in the Dartmouth experiment [1]. Consequently the radiation occurs in the $\mathrm{THz}$ regime. However, the grating length in our simulation is shorter than the one used in the Dartmouth experiment because of the limited capacity of our computers. In addition, the form of the beam is different, since we use a sheet beam and the experiment used a round beam.

As to the diagnostics, MAGIC allows us to observe a variety of physical quantities such as electromagnetic fields as functions of time and space, power outflow, and electron phase-space trajectories [19]. We can set the relevant detectors anywhere in the simulation area.

\section{SIMULATION RESULTS}

\section{A. A single bunch}

In this simulation, we make a single electron bunch pass over a grating having 20 periods. The electron bunch is generated with a rectangular profile and a length of $0.1 \mathrm{ps}$. We choose the beam current as $480 \mathrm{~mA}$ and hence the bunch has the charge of $0.048 \mathrm{pC}$. The bunch length is small compared to the SP radiation wavelength given by Eq. (1), so the radiation is coherent. We run the code for enough time to ensure that the main phenomena appear and, especially, the radiation emitted can reach the detector.

The process of radiation generation can be observed through the contour plots of the magnetic field $B_{z}$. We give four plots in the time order as shown in Figs. 2(a)2(d), where one can clearly see the crescent-shaped wave fronts of the radiation. Figure 2(a) shows that the bunch has covered 10 periods of the grating and come to the center, and 10 crescents appear in the vacuum area, which means the electron bunch radiates at every period of grating. Together with the frequency property given later, we recognize that this is SP radiation. In Fig. 2(b), one can observe that the electron bunch has covered all the periods and arrived at the end of the grating. Figure 2(c) is snapped 

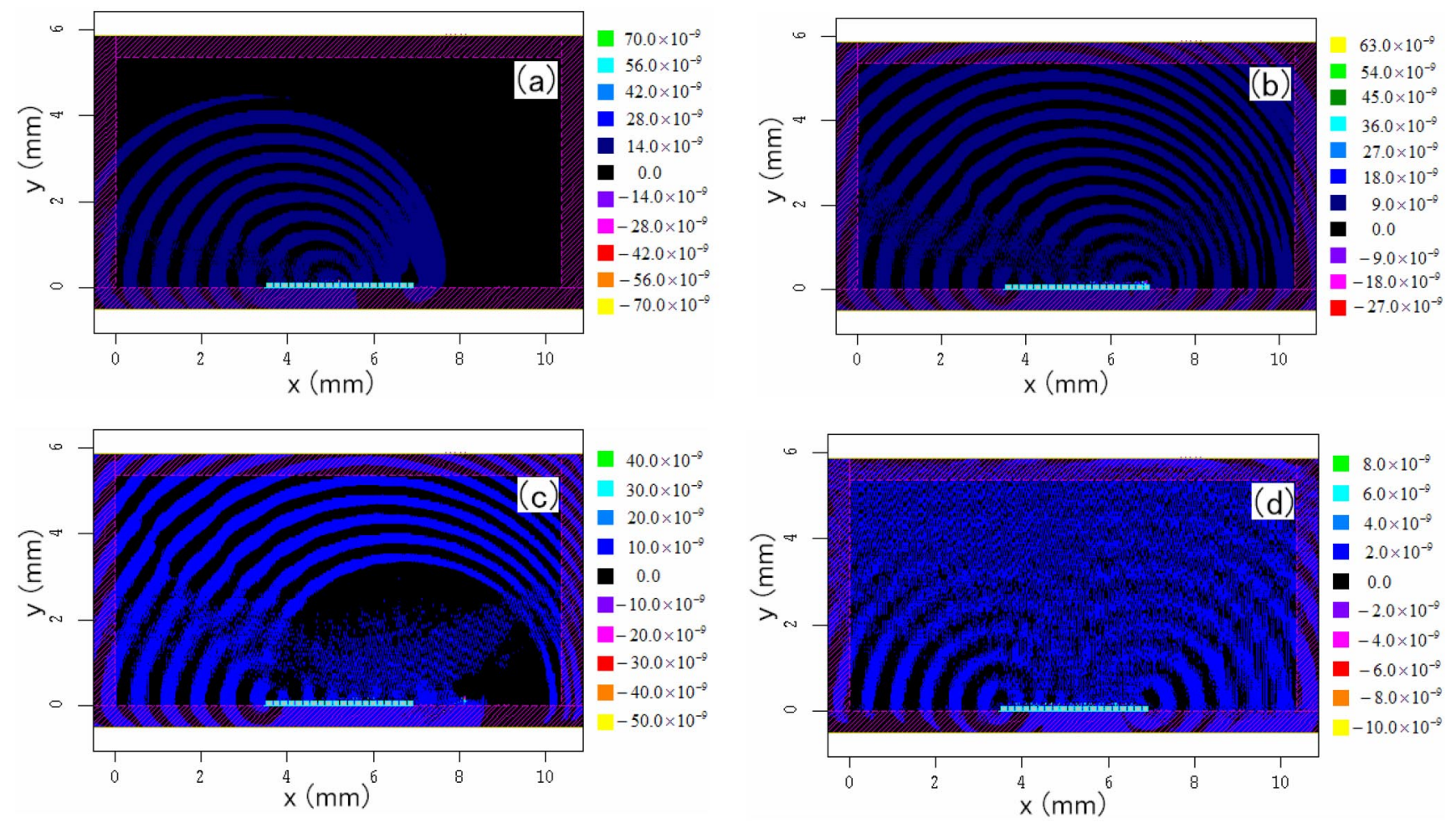

FIG. 2. (Color) Contour plots of $B_{z}$ at (a) $t=50.9 \mathrm{ps,} \mathrm{(b)} t=65.6 \mathrm{ps,} \mathrm{(c)} t=77 \mathrm{ps,} \mathrm{(d)} t=148.9 \mathrm{ps}$.

at the time when the bunch has moved beyond the grating, and we notice that the SP radiation is no longer being emitted and a blank appears in the plot. But at a later time, as shown in Fig. 2(d), an interesting thing happens. We see that two cylindrical waves radiated from both ends of the grating appear and form a clear interference pattern. We deduce that those waves should be attributed to the evanescent wave radiation. The evanescent wave does not radiate until it reaches the ends of a grating, where it undergoes partial reflection and partial diffraction [15].

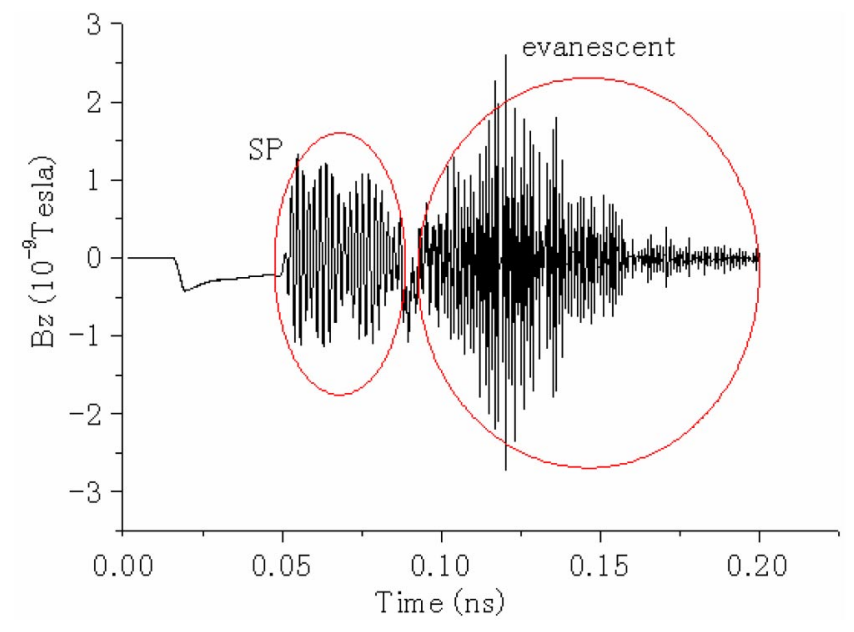

FIG. 3. (Color) Time signal of $B_{z}$, observed at point $5.346 \mathrm{~mm}$ and $130^{\circ}$ from the center of the grating.
The reflected portion then oscillates within the grating and keeps radiating at both ends, which should be the reason that long after the electron bunch has left the simulation area the radiation still comes out. In addition, the group velocity of the evanescent wave is slow, about one-fifth the light velocity [15] under the present situations, which is the reason for the appearance of the blank between SP and evanescent wave emissions.

The $B_{z}$ detector is placed at the distance $5.346 \mathrm{~mm}$ from the grating center. The temporal behavior observed at $130^{\circ}$ is given in Fig. 3, where we see the 20 periods of the SP radiation and the oscillation of the evanescent wave. And it is clearly shown that the SP radiation is separated in time from the evanescent wave. The corresponding fast-Fouriertransform (FFT) of Fig. 3 is shown in Fig. 4, which demonstrates the SP radiation and the evanescent wave signals, respectively. The center frequency of the SP radiation is $525 \mathrm{GHz}$, corresponding to the basic SP equation. The evanescent wave frequency is $445 \mathrm{GHz}$, independent of radiation angle and lower than the minimum allowed SP radiation frequency. This result is in agreement with the viewpoint of Andrews and co-workers $[15,16]$. We vary the observation direction and get the dependence of the SP radiation frequency on the radiation angle as shown in Fig. 5. The simulation result is very close to the theoretical curve, with typical discrepancies about $1 \%$. Also plotted in Fig. 5 is the distribution of $B_{z}$ amplitude. It shows that the radiation is weak at small angle and maximizes at $125^{\circ}$ for this simulation. The amplitude of the evanescent wave is 


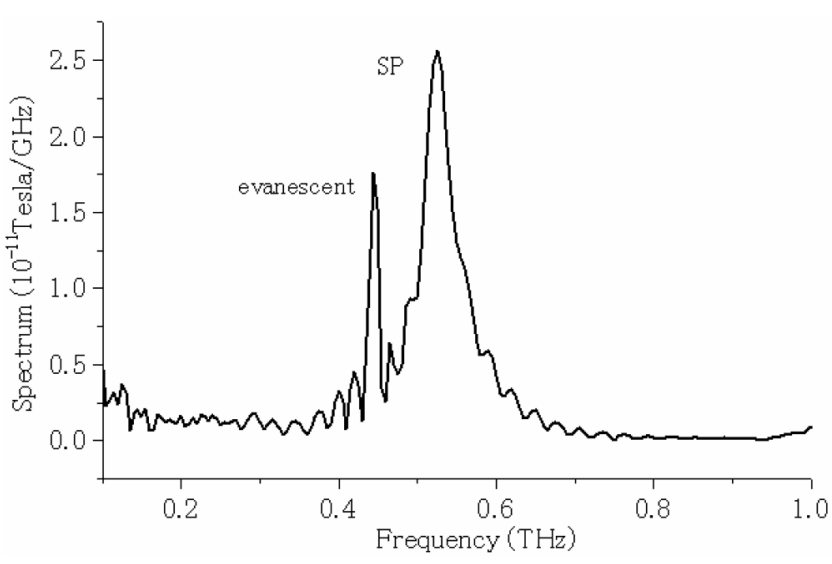

FIG. 4. FFT of time signal corresponding to Fig. 3.

not plotted for clarity. Hereafter, we concentrate only on the SP radiation.

\section{B. Train of bunches}

In all radiation sources using an intense electron beam, the mechanism leading to superradiance is beam bunching. The spectral intensity of the radiation will be enhanced at the bunching frequency and its harmonics. The superradiant SP radiation has been demonstrated in recent experiments, which employed a prebunched electron beam and an initially continuous beam, respectively. Korbly and coworkers carried out a SP experiment at MIT [20], by producing a train of bunches with an accelerator before it is introduced in the grating, and they observed the superradiance emitted at harmonics of the bunching frequency. The continuous beam in a grating system could be bunched by the interaction with the evanescent wave when proper conditions are satisfied and reach the superradiance, like the Dartmouth experiment [1]. In order to demonstrate the properties of superradiant radiation more clearly, we avoid

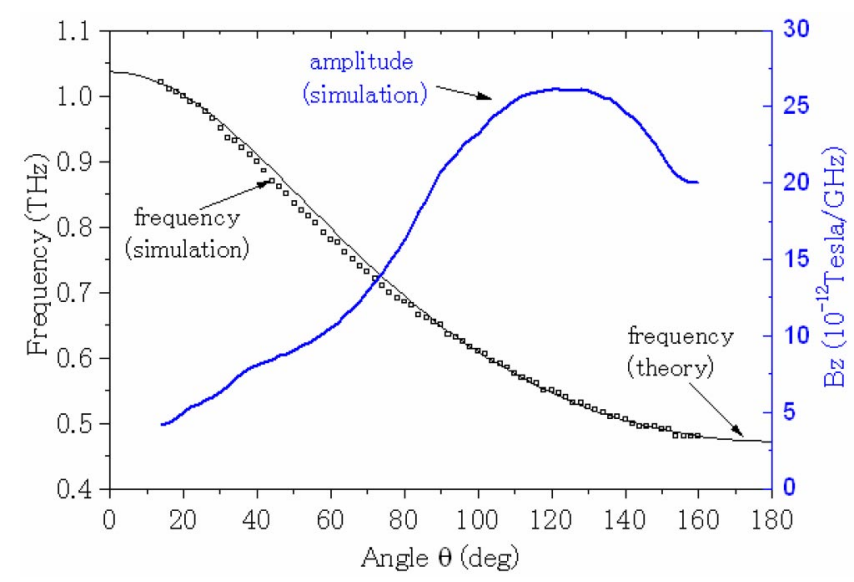

FIG. 5. (Color) Radiation frequency and the peak of FFT amplitude of $B_{z}$ as a function of angle, detected at the distance $5.346 \mathrm{~mm}$ from the grating center.

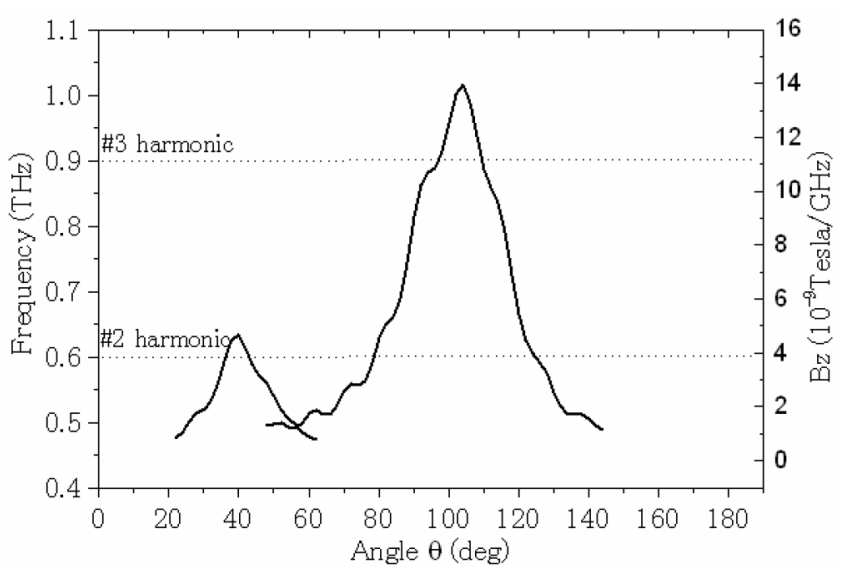

FIG. 6. Radiation frequency and the peak of FFT amplitude of $B_{z}$ of superradiant radiation as a function of angle, detected at the distance $5.346 \mathrm{~mm}$ from the grating center.

the problem of bunching from an initially continuous beam. Instead, we generate a train of bunches to drive the grating.

We again use a grating having 20 periods. The repetition frequency of bunches is set to be $300 \mathrm{GHz}$, which is different from the evanescent wave frequency. The parameters for each single bunch are the same as those mentioned earlier. Within the time of code running, 60 bunches are produced and enter the simulation area. From the FFT of the temporal behavior observed by the $B_{z}$ detectors we know that the radiation is concentrated on two frequencies, the second and the third harmonic of bunching frequency, which are both allowed frequencies of the first order SP radiation, as illustrated in Fig. 6. The dominant radiation is the second harmonic, which peaks at the angle of $104^{\circ}$ corresponding to the value determined by Eq. (1). The other one radiates at the third harmonic with the angle of about $40^{\circ}$. The phenomenon that superradiant SP radiation is emitted at a certain direction can be observed not only in Fig. 6, but also in the contour plot of Fig. 7. From the contour plot, one can easily understand that the dominant second harmonic radiates at the angle of about $104^{\circ}$, in agreement with what is shown in Fig. 6 .

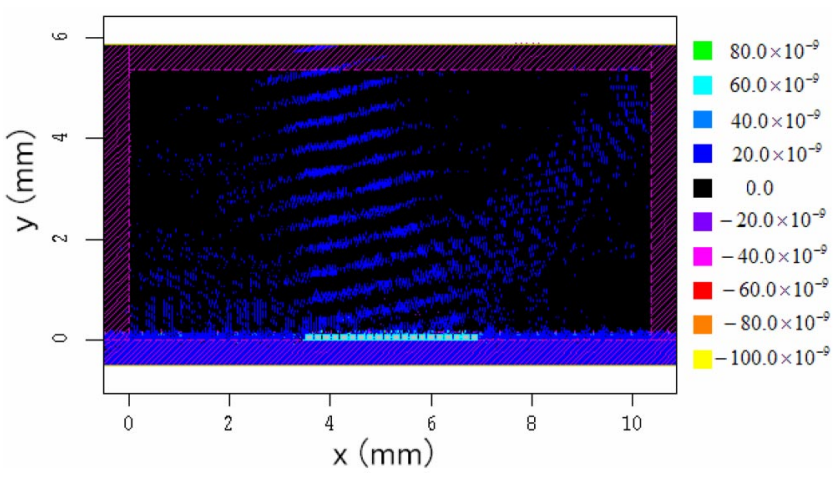

FIG. 7. (Color) Contour plot of $B_{z}$ for superradiant radiation. 
Recently, Kesar and co-workers reported the power measurement of SP radiation excited by a train of electron bunches [21]. Their measurements support our finding. It also should be noted that, Andrews and co-workers predicated the frequency characteristics of the superradiant SP radiation in their theoretical analysis. Our simulation results conform to their viewpoint.

\section{Continuous beam}

As we mentioned earlier, Donohue and Gardelle have addressed the evanescent wave, electron beam bunching and the radiation gain, and described the characteristics of the superradiant radiation through the simulation of a continuous beam. Here, we concentrate only on demonstrating the relation of the output power of SP radiation and the beam current, which is an experimental result of Dartmouth.

In the Dartmouth experiment, a grating consisting of 73 periods was employed. We cannot use the same number of periods in our simulation due to the limit of the capacity of our computers, instead, 50 periods are used. For this simulation, the electron beam from the cathode is continuous, and an external magnetic field of $2 \mathrm{~T}$ is introduced to prevent the beam from diverging. We vary the beam current and observe the total power flow out of the top plane. From the theoretical analysis in Ref. [15], we know that the beam line intersects the dispersion curve at a point representing a backward wave, which means the device operates in the mode of backward-wave oscillator (BWO). Such a device can start to oscillate without external feedback when the beam current exceeds a threshold value. During the simulation, we found that there surely exists a certain value for the beam current, above which the evanescent wave becomes strong enough to induce the beam bunching and in turn the bunched beam excites the superradiant SP radiation as discussed above. We read the peak power of SP radiation through the observation of power spectrum, and plot the result in Fig. 8, where we can easily identify two regimes for incoherent and superradiant radiation, respectively. The turn point for the two regimes gives the threshold value for the present simulation. This result also supports the theory reported in a very recent paper of Andrews and co-workers [22]. Because of the differences in electron beam model and the grating length, our simulation is not comparable with the Dartmouth experiment. Nevertheless, we find that Fig. 8 resembles the experimental result in Ref. [1].

The frequencies for the two regimes are different, as shown in Fig. 9. The incoherent regime shows a value of about $650 \mathrm{GHz}$, which is close to the frequency of an ordinary SP radiation emitted at $90^{\circ}$. The superradiant regime gives a value of about $890 \mathrm{GHz}$, which is the second harmonic of the evanescent wave. According to the property of superradiance analyzed earlier, we know the superradiant radiation of this frequency should be emitted

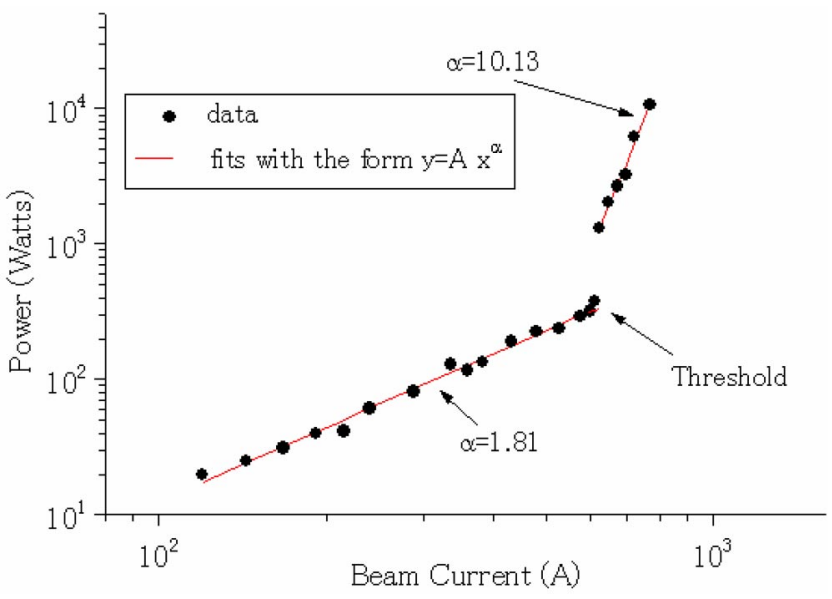

FIG. 8. (Color) Output power of SP radiation with respect to the beam current.

mainly at the angle of $45^{\circ}$. Fortunately, with our simulation geometry the power detector placed on the top plane can catch a portion of this radiation, and so we get the result as shown in Fig. 8. Figure 9 shows that the frequency slightly decreases as the beam current increases. This is due to the space charge reducing the electrons' velocity, which has been also observed in Ref. [18].

We can simply understand the influence of the space charge like this. A static electric field pointing from the grating to the beam exists when the current is steady, and this field leads to a negative electrostatic potential in the position of beam. If the beam is supposed to be thin and at a distance $\delta$ from the grating, the average potential can be estimated as $V=I \delta /\left(\varepsilon_{0} v\right)$ by using Gauss's law. Here, $I$ denotes the current/meter, $\varepsilon_{0}$ the permittivity of free space and $v$ the velocity of the beam. The electrons in the beam thus have positive potential energy meaning that their kinetic energy is $T_{0}-|V|$ (in $\mathrm{eV}$ ), where $T_{0}$ is the initial kinetic energy.

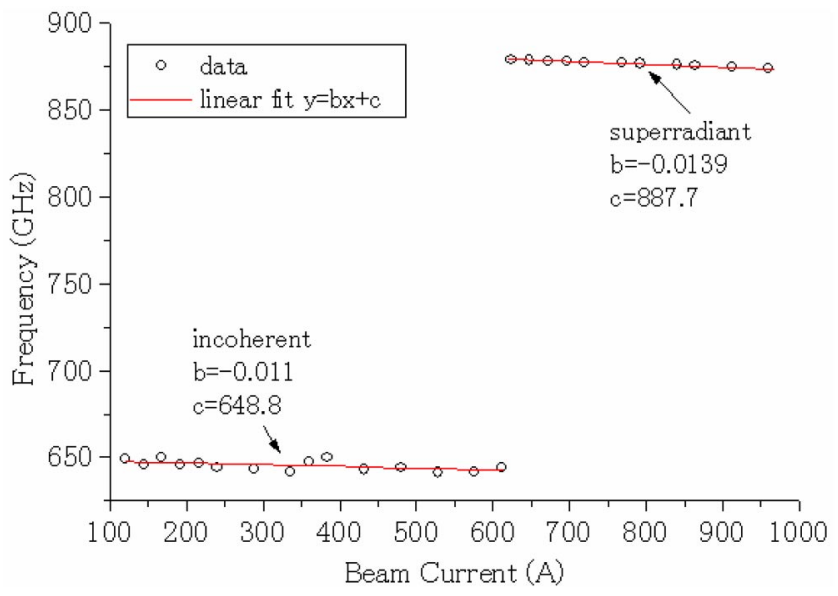

FIG. 9. (Color) Frequencies of incoherent and superradiant radiation with respect to beam current. 
The velocity reduction influences the frequency of incoherent and superradiant radiation in different ways: for the case of incoherent radiation, the lower velocity will give rise to lower frequency corresponding to Eq. (1); while for the case of superradiant radiation, the reduction of particle velocity makes the intersection of beam line and dispersion curve shift to smaller frequency, which means a decrease of the frequency of the evanescent wave.

\section{CONCLUSION}

In conclusion, we have studied the coherent and superradiant SP radiation through simulation of an open grating system driven by different modes of electron beam. The single bunch simulation helps us to distinguish the true SP radiation from the evanescent wave. They are different in both frequency characteristics and generation mechanism. Under the present situations, we find that the SP radiation is emitted just as the electron bunch flies over the grating, whereas the evanescent wave can oscillate and keep diffracting from both ends of the grating even after the bunch has left. The amplitude of the SP radiation is angle dependent. The strongest radiation appears at $125^{\circ}$ at the present parameters. The supperandiant effect is demonstrated with the simulation of a prebunched beam. We provide powerful evidence showing that the superradiant radiations are emitted at frequencies that are integer multiples of the bunching frequency, and at the corresponding SP direction, which strongly support the theory of Andrews and co-workers. The simulation of a continuous beam provides us the relation between output power and the beam current, which resembles the result of Dartmouth experiment. Urata and co-workers mentioned in their paper that no discernible difference in the frequency between the incoherent and the superradiant regimes were observed by their detection. However, our simulations show an apparent difference, and we point out that the radiations from the two regimes are due to different physical mechanisms. The incoherent regime follows the properties of ordinary SP radiation, so that the radiation frequency observed from the top plane is close to the frequency emitted at $90^{\circ}$, whereas the superradiant frequency strongly depends on the bunching frequency, which is the evanescent wave frequency in the case of using a continuous beam.

\section{ACKNOWLEDGMENTS}

The authors gratefully acknowledge useful discussions with John Donohue, Charles Brau, and Heather Andrews.

[1] J. Urata, M. Goldstein, M.F. Kimmitt, A. Naumov, C. Platt, and J.E. Walsh, Phys. Rev. Lett. 80, 516 (1998).

[2] A. Bakhtyari, J. E. Walsh, and J. H. Brownell, Phys. Rev. E 65, 066503 (2002).

[3] S. P. Mickan and X.-C. Zhang, Int. J. High Speed Electron. Syst. 13, 601 (2003).

[4] P. H. Siegel, IEEE Trans. Microwave Theory Tech. 50, 910 (2002).

[5] S. J. Smith and E. M. Purcell, Phys. Rev. 92, 1069 (1953).

[6] P. M. van den Berg, J. Opt. Soc. Am. 63, 689 (1973).

[7] P. M. van den Berg and T. H. Tan, J. Opt. Soc. Am. 64, 325 (1974).

[8] L. Schachter, Beam-Wave Interaction in Periodic and Quasi-Periodic Structures (Springer-Verlag, Berlin, 1996).

[9] Yu. N. Dnestrovskii and D. P. Kostomarov, Sov. Phys. Dokl. 4, 158 (1959).

[10] Y. Takakura and O. Haeberlé, Phys. Rev. E 61, 4441 (2000).

[11] J. Walsh, K. Woods and S. Yeager, Nucl. Instrum. Methods Phys. Res., Sect. A 341, 277 (1994).

[12] Y. Shibata, S. Hasebe, K. Ishi, S. Ono, M. Ikezawa, T. Nakazato, M. Oyamada, S. Urasawa, T. Takahashi, T. Matsuyama, K. Kobayashi, and Y. Fujita, Phys. Rev. E 57, 1061 (1998).

[13] L. Schachter and A. Ron, Phys. Rev. A 40, 876 (1989).

[14] K. -J. Kim and S. -B. Song, Nucl. Instrum. Methods Phys. Res., Sect. A 475, 158 (2001).

[15] H. L. Andrews and C.A. Brau, Phys. Rev. ST Accel. Beams 7, 070701 (2004).

[16] H.L. Andrews, C.H. Boulware, C.A. Brau and J.D. Jarvis, Phys. Rev. ST Accel. Beams 8, 050703 (2005).

[17] A. S. Kesar, M. Hess, S. E. Korbly, and R. J. Temkin, Phys. Rev. E 71 , 016501 (2005).

[18] J. T. Donohue and J. Gardelle, Phys. Rev. ST Accel. Beams 8, 060702 (2005).

[19] L. Ludeking, "The MAGIC User's Manual."

[20] S. E. Korbly, A. S. Kesar, J. R. Sirigiri, and R. J. Temkin, Phys. Rev. Lett. 94, 054803 (2005).

[21] Amit S. Kesar, Roark A. Marsh, and Richard J. Temkin, Phys. Rev. ST Accel. Beams 9, 022801 (2006).

[22] H. L. Andrews, C.H. Boulware, C. A. Brau, and J.D. Jarvis, Phys. Rev. ST Accel. Beams 8, 110702 (2005). 